\title{
Investigation of Compressibility Effect for Aeropropulsive Shear Flows
}

\author{
M. S. Balasubramanyam ${ }^{*}$ and C.P. Chen ${ }^{\S}$ \\ University of Alabama in Huntsville, Huntsville, AL 35899
}

\begin{abstract}
Rocket Based Combined Cycle (RBCC) engines operate within a wide range of Mach numbers and altitudes. Fundamental fluid dynamic mechanisms involve complex choking, mass entrainment, stream mixing and wall interactions. The Propulsion Research Center at the University of Alabama in Huntsville is involved in an ongoing experimental and numerical modeling study of non-axisymmetric ejector-based combined cycle propulsion systems. This paper attempts to address the modeling issues related to mixing, shear layer/wall interaction in a supersonic Strutjet/ejector flow field. Reynolds Averaged Navier-Stokes (RANS) solutions incorporating turbulence models are sought and compared to experimental measurements to characterize detailed flow dynamics. The effect of compressibility on fluids mixing and wall interactions were investigated using an existing CFD methodology. The compressibility correction to conventional incompressible twoequation models is found to be necessary for the supersonic mixing aspect of the ejector flows based on 2-D simulation results. 3-D strut-base flows involving flow separations were also investigated.
\end{abstract}

\section{INTRODUCTION}

\begin{abstract}
A fundamental understanding of ejector flow physics is an enabling technology to realize an operational Rocket Based Combined Cycle (RBCC) propulsion system. Past theoretical and experimental ejector studies have considered one-dimensional, axisymmetric, or at best two-dimensional geometries. Concepts such as the Strutjet use a complex asymmetric, three-dimensional flow path. The Propulsion Research Center (PRC) of University of Alabama in Huntsville (UAH) has an ongoing research program to characterize asymmetric ejector performance in terms of mass flow entrainment, stream mixing, and choking mechanisms using both an experimental approach and computational fluid dynamics (CFD) modeling approach ${ }^{1}$

Validation of computational fluid dynamic (CFD) models appropriate for supersonic through hypersonic flow applications requires careful consideration of the physical processes encountered at these flight regimes and detailed comparisons of the calculated results with experimental data sets that include these processes. Supersonic ejectors perform the mixing and recompression of the primary flow (ejector) and the secondary (induced) flow ${ }^{2}$. The primary flow gets accelerated by a convergent divergent nozzle to reach supersonic velocity. By an entrainment-induced effect, the secondary stream is drawn into the flow and accelerated. Mixing and recompression of the resulting stream then occurs in a mixing chamber, where complex interactions take place between the mixing layer and shocks. In Reference 1, several turbulence models ranging from the algebraic to two-equation models were systematically evaluated for 2-D single-nozzle ejector flow field. Depending on the operating conditions of the ejector chamber pressure, the mixing patterns and the shear layer/wall interactions exhibit drastic changes. In general, it was concluded that the SST two-equation model of Menter gave the most satisfactory results ${ }^{1}$. The SST model is a combination of two popular two-equation models: the $k-\omega$ and the $k-\varepsilon$ models. A blending function was devised such that $k$ - $\omega$ model was used in the wall region and a switch to the $k-\varepsilon$ model occurred in the outer wake/free stream region. In supersonic mixing regime, the $k-\varepsilon$ model was found to over-predict the mixing of compressible shear layers ${ }^{3}$. Various compressibility corrections to the $k-\varepsilon$ model was proposed [see Chapter 5 of Reference 4], and the performance of these models were inconclusive.
\end{abstract}

\footnotetext{
- Graduate Research Assistant, Student Member AIAA

$\S$ Professor, Department of Chemical and Materials Engineering, Senior Member AIAA.

This material is a work of the U.S. Government and is not subject to copyright protection in the United States.
} 
For the flow field encountered in the present study, it was not clear what role compressibility corrections would play. The purpose of this paper is to incorporate an existing compressibility correction into the $k-\varepsilon$ model, for calculating mixing patterns of ejector flows under various operating conditions. In addition, the 3-D features of the investigated flow will be discussed and modeled.

\section{NUMERICAL MODELING}

Compressibility effects, which reduce mixing and turbulent intensity are present in supersonic free shear aeropropulsive flows and can be significant for large convective Mach numbers. A parameter that could be used to characterize the effect of compressibility on the turbulent fluctuations is the turbulent Mach number, which is defined as the ratio of a characteristic speed of turbulence to the wave speed. Thus $M_{t}=\sqrt{ } 2 k / a$, where ' $k$ ' is the turbulent kinetic energy and ' $a$ ' is the speed of sound. As pointed by various researchers ${ }^{5,6}$ the standard $k$ - $\varepsilon$ model fails to predict the observed decrease in spreading rate for the compressible free shear layers. Compressibility is modeled using dilatational and pressure fluctuation terms within the turbulence quantity conservation equations. Concentrating on the $k$ - $\varepsilon$ model, the dissipation of the kinetic energy in compressible turbulence was argued to be augmented by the dilatational components $\varepsilon_{\mathrm{d}}$ of the dissipation tensor in addition to the solenoidal components. Sarkar ${ }^{7}$ et al., considered the evolution of the turbulence fluctuations on an acoustic time scale. They considered the effect of varying compressibility based on the turbulent Mach number $\mathrm{M}_{\mathrm{t}}$, on the rate of dissipation of turbulent kinetic energy, $\varepsilon$. Analysis of decaying compressibility on the solenoidal component of the dissipation rate, $\varepsilon_{\mathrm{s}}$, was negligible in comparison with the effect on the dilatational component, $\varepsilon_{\mathrm{d}}$. They also observed that the ratio of dilatational dissipation rate to the solenoidal dissipation rate varied directly as the square of the turbulent Mach number $\mathrm{M}_{\mathrm{t}}$. They proposed a model for the dilatational dissipation rate, which is given as

$$
E_{d}=\alpha_{1} \varepsilon_{s} M_{t}^{2}, \quad \text { where } \alpha_{1} \text { is an arbitrary constant of order } 1 .
$$

Adding the above term would account for the compressibility correction in the conventional two-equation model. The baseline two-equation model used in this study is the standard $k-\varepsilon$ model, written for mass weighted turbulence quantity for turbulence kinetic energy ' $k$ ' with the extra compressibility term being:

$$
\frac{\partial}{\partial t}(\rho k)+\frac{\partial}{\partial x_{j}}\left(\rho u_{j} k\right)=P-\rho \varepsilon+\frac{\partial}{\partial x_{j}}\left[\left(\mu+\frac{\mu_{t}}{\sigma_{k}}\right) \frac{\partial k}{\partial x_{j}}\right]-\alpha_{1} \frac{k}{a^{2}} \rho \varepsilon
$$

where $\alpha_{1}=2.0$, and its dissipation rate ' $\varepsilon$ ' are

$$
\frac{\partial}{\partial t}(\rho \varepsilon)+\frac{\partial}{\partial x_{j}}(\rho u, \varepsilon)=C_{\varepsilon_{1}} \frac{P \varepsilon}{k}-C_{\varepsilon_{2}} \frac{\rho \varepsilon^{2}}{k}+\frac{\partial}{\partial x_{j}}\left[\left(\mu+\frac{\mu_{i}}{\sigma_{k}}\right) \frac{\partial \varepsilon}{\partial x_{j}}\right]
$$

Sarkar et al. ${ }^{7}$ and Zeman ${ }^{5}$ have postulated that the transport equation for $\varepsilon$ is unaffected by compressibility. The compressibility corrections were incorporated into the density-based CFD-FASTRAN ${ }^{\circledR} \operatorname{code}^{8}$, for the $k-\varepsilon$ turbulence model.

\section{RESULTS AND DISCUSSION}

Before modeling the supersonic ejector flows, the implemented compressibility model was first validated with the supersonic backward-facing benchmark case of Eklund et al ${ }^{9}$. It should be stated that grid-refinement studies were carried out ${ }^{10}$ and the results presented here are grid-independent.

\section{Supersonic Backward Facing Step}

The Mach 2 flow over a backward-facing step has been investigated experimentally using a variety of techniques. The test section geometry and the reattachment length calculations are shown in Table 1 . The flow field contains many complex flow features including boundary layer separation, reattachment, viscous shear layer and expansion fan/shock wave interaction. In the calculation, the expansion fan, centered on the step, turns the flow over 
the step. Behind the step a recirculating region of low-momentum fluid that is characterized by low velocities and low pressures is formed. Immediately behind the step a shear layer forms between the accelerating flow turning over the step and the recirculating flow. Downstream of the step a reattachment shock turns the flow parallel to the wall.

The net effect of the compressibility correction is to reduce the magnitude of turbulence kinetic energy, thus the effective eddy viscosity, and to delay the flow reattachment. Both the standard $k-\varepsilon$ and the compressibility corrected $k-\varepsilon$ model utilized the wall-function approach in this study. The SST model of Menter utilized $k-\omega$ blending for near wall treatment and recovered the standard $k-\varepsilon$ model at the outer region. Thus the SST model is viewed as the wallresolving $k-\varepsilon$ model without compressibility correction. Figures $1 \mathrm{a}$ and $1 \mathrm{~b}$ show the calculated velocity profiles (normalized with respect to mean inlet velocity) at the upstream and downstream locations of the reattachment. As can be seen from the figures, due to the reduction of shear layer mixing, the compressibility correction gave longer

Table 1. Test Geometry \& Reattachment Lengths

\begin{tabular}{lclc}
\hline Feature & Dimension & & Reattachment Length \\
\hline Step Height & $\mathrm{H}=0.00318 \mathrm{~m}$ & Experimental & $3.9 \mathrm{H}$ \\
Test Section Height & $6.71 \mathrm{H}$ & Menter SST & 3.20 \\
Test Section Length & $10 \mathrm{H}$ & Standard $k-\varepsilon$ & 3.65 \\
& & $k-\varepsilon /$ compress. & 3.81
\end{tabular}

reattachment length and compared better with experimental data. The SST model, though it was originally based on $k$ - $\omega$ compressible flow formulation, did not have explicit compressibility correction at shear layer region and underpredicts the reattachment length.

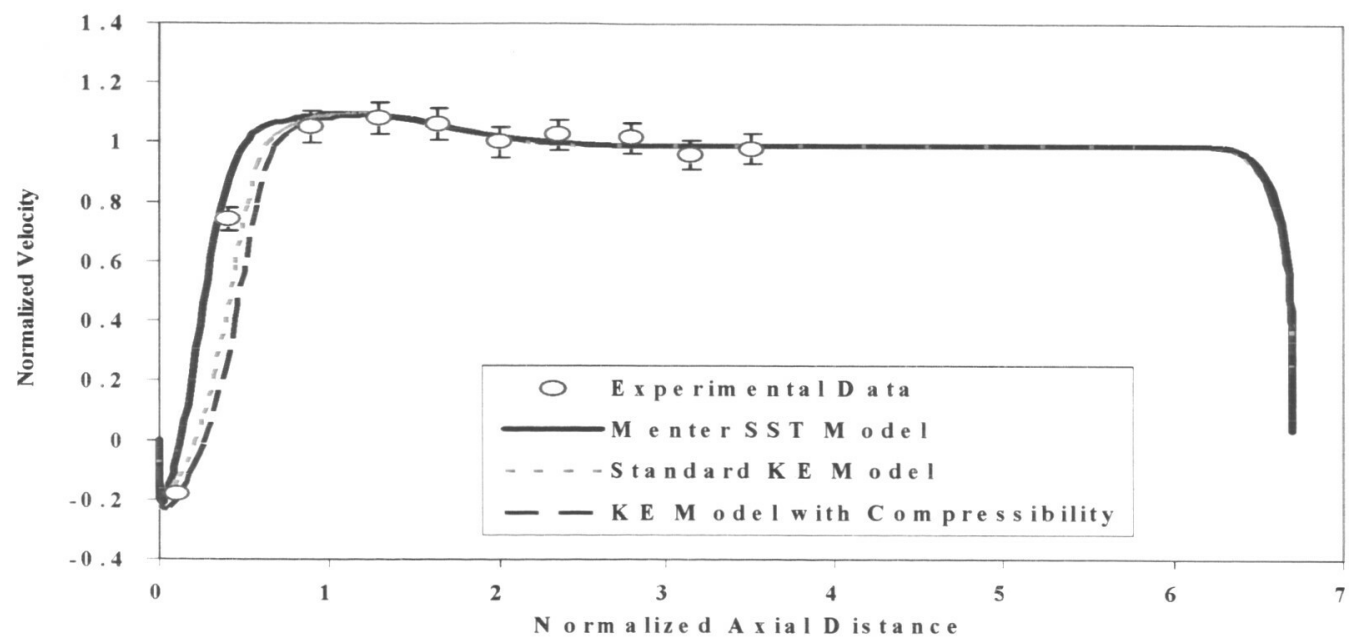

Figure 1a. Normalized velocity profiles at $\mathrm{X}=1.7 \mathrm{H}$ (recirculation region)

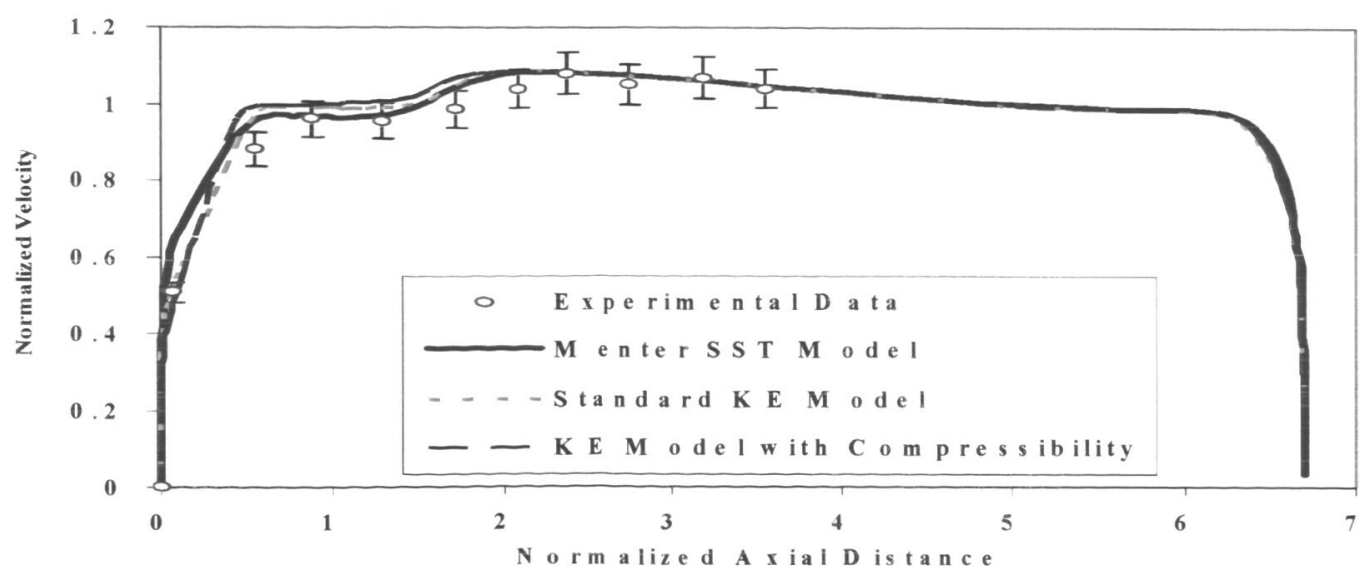

Figure $1 \mathrm{~b}$. Normalized velocity profiles at $\mathrm{X}=61.7 \mathrm{H}$ (downstream region)

3

American Institute of Aeronautics and Astronautics 


\section{UAH's Strutjet Ejector}

As described in Lineberry et al., ${ }^{11}$ the single-nozzle asymmetric ejector flow system involves complicated phenomena including shock-boundary layer interaction, mass choking, jet mixing and secondary flow separations. In Reference 1, the ejector mass choking phenomena created by a high-pressure jet within confined environment, as well as the mixing patterns were studied. The compressibility effect of interest here is applied to the mixing region (see Figure 2). Due to the large aspect ratio geometry of the single-strut configuration, it was beneficial to carry out detailed 2-D simulations (see also Reference 1) to first investigate the relative performance of the turbulence model with compressibility correction. The sidewall database was chosen for this purpose. The ejector geometry, grid systems, as well as the boundary condition specifications were described in Reference 1 .

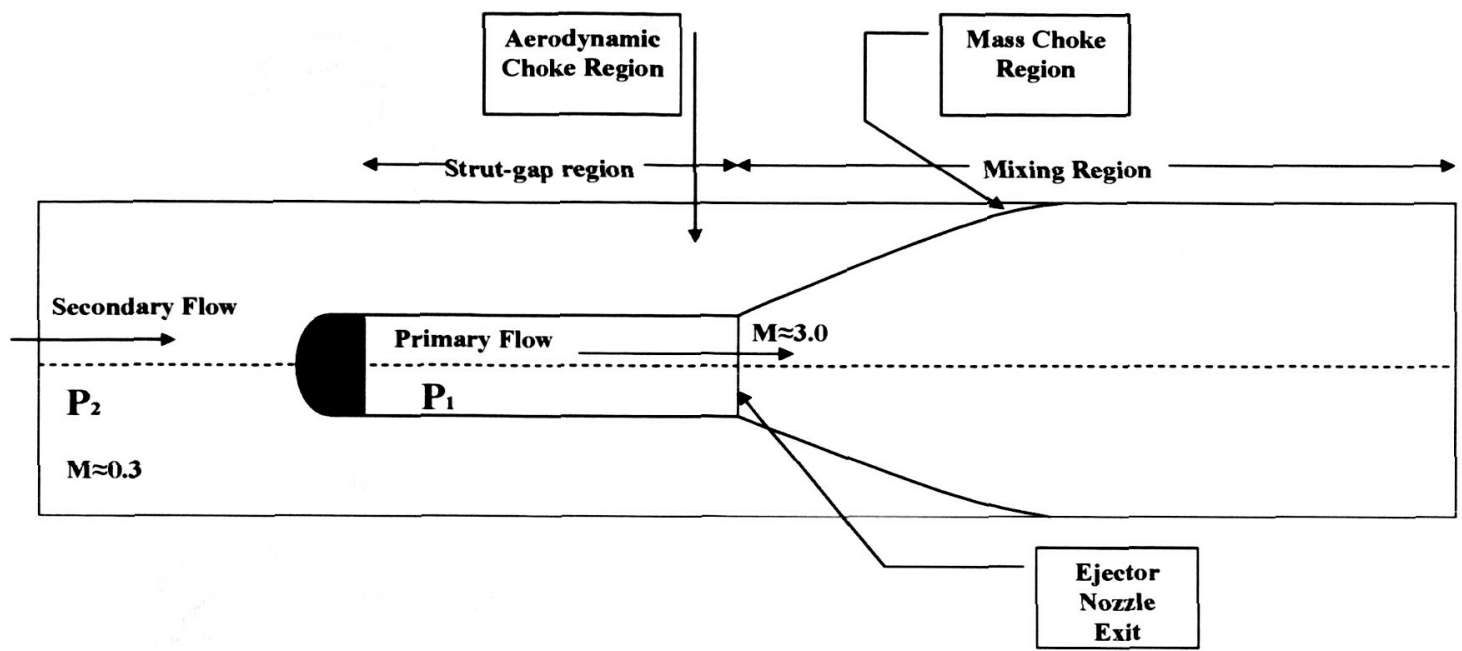

Figure 2. Strut-jet ejector flow regions

Figures 3 to 5 show the comparison of calculated side-wall pressures with the experimental data for three different strutjet chamber pressures. The $k$ - $\varepsilon$ model with and without compressibility corrections, and the SST model were used in these CFD calculations. As pressure level of the ejector changes, the flow pattern changes drastically. At a chamber pressure of $600 \mathrm{psi}$, the mixing length was about $0.88 \mathrm{~m}$, from experimental data. The complete mixing of the primary and secondary streams is indicated when the side wall pressures recover to the external condition, i.e. one ATM.

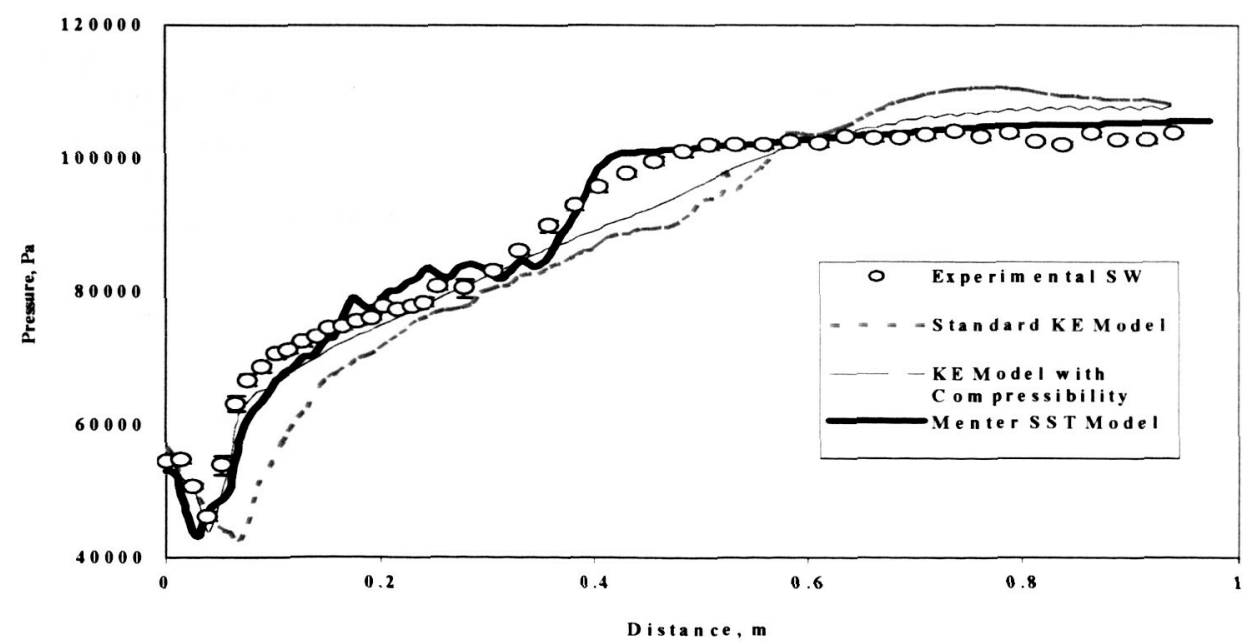

Figure 3. Side-wall pressure comparisons for ejector chamber pressure at $600 \mathrm{psi}$ 


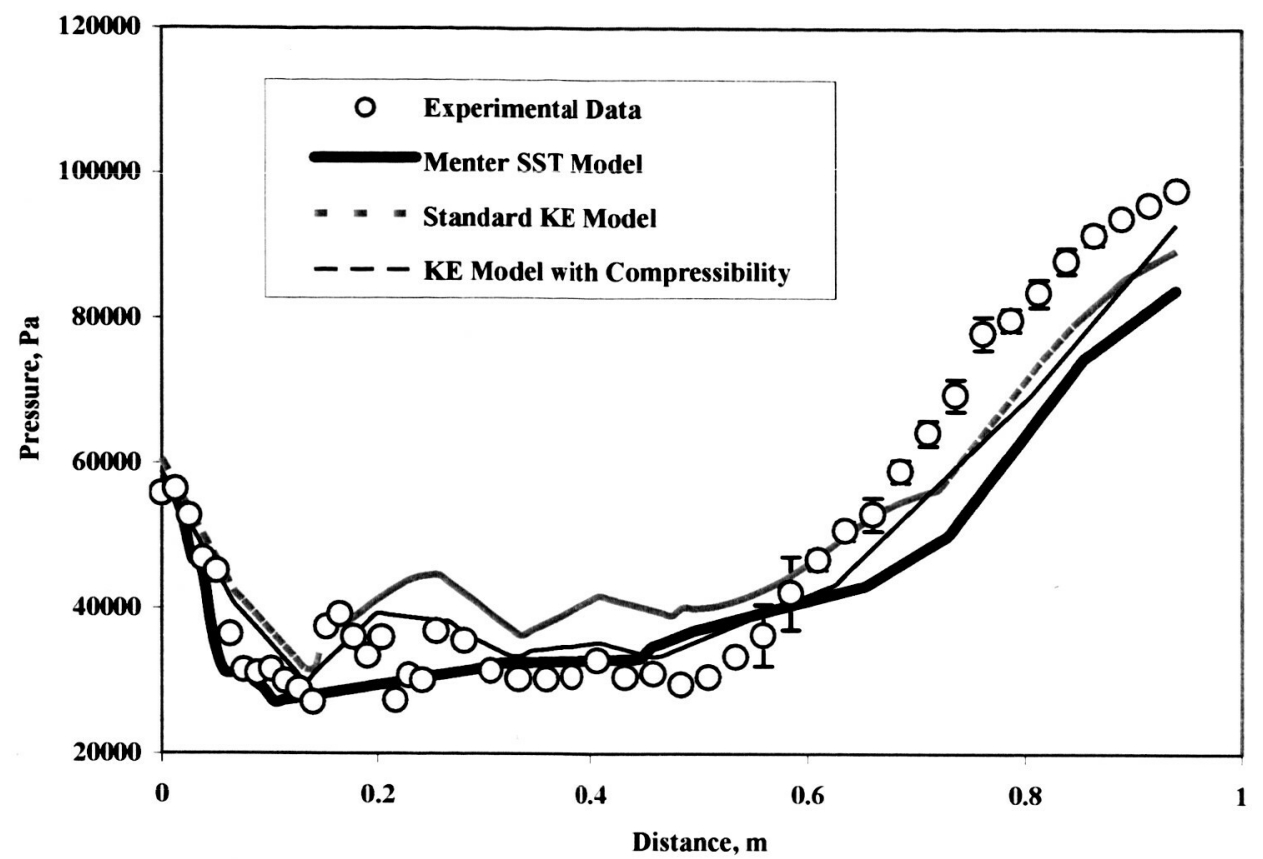

Figure 4. Side-wall pressure comparisons for ejector chamber pressure $700 \mathrm{psi}$.

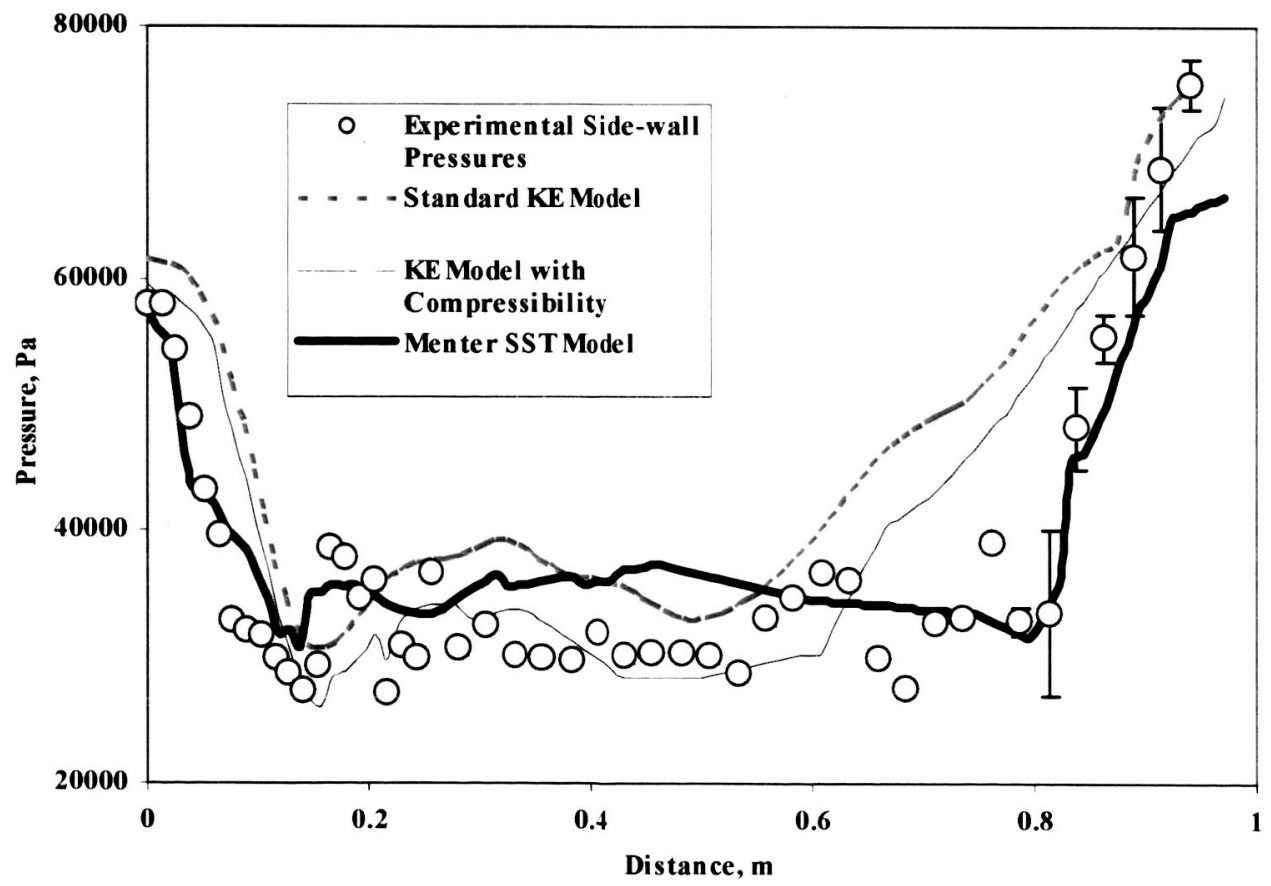

Figure 5. Side-wall pressure comparisons for ejector chamber pressure $800 \mathrm{psi}$. 
From these figures, it can be seen that within the mixing zone, the compressibility corrected $k$ - $\varepsilon$ model shows much improved predictions when compared with the standard $k-\varepsilon$ model and experimental data. However, after the complete mixing zone, the wall effect becomes dominant, and the wall function approach used in both $k$ - $\varepsilon$ models (with and without compressibility correction) cannot capture the pressure recovery. On the other hand, the SST model performs well in the recovery regions due to its use of $k$ - $\omega$ formulation at the wall region as seen clearly in Figures 3 and 5.

\section{Three Dimensional Flow Characteristics}

One flow characteristic associated with the non-axisymmetric ejector flow field is the three-dimensionality in the strut-base region. As depicted in Figure 6, as the primary stream is accelerated through a convergent-divergent nozzle to a supersonic Mach 3 jet, the secondary stream flows through the strut gap and is then entrained by the primary flow. When the secondary flow tries to expand around the corner as it passes the strut, a separation region will be formed, characterized by low static pressures. The experimental study of Reference 11 also supplied pressure distribution data at the top wall. A full 3-D CFD calculation is required to capture this flow feature. Preliminary results using the wall-resolved Baldwin-Lomax turbulence model is shown in Figure 7. The good agreement between the prediction and data before $0.1 \mathrm{~m}$ and the ability of capturing the flow separation by the Baldwin-Lomax model indicated that turbulence models capable of resolving near wall regions should be

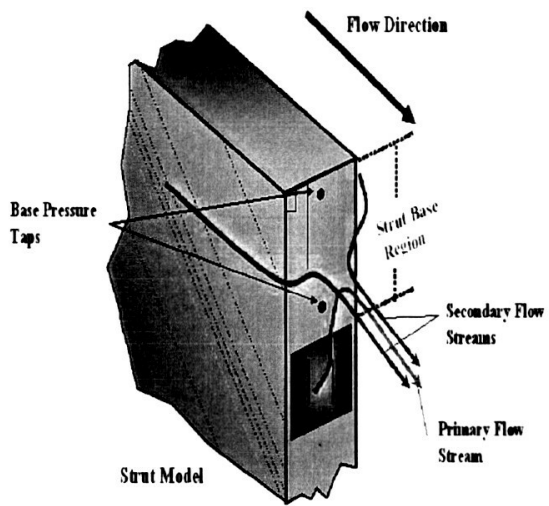

Figure 6. Strut-base separation region used for this 3-D ejector flow field. Compressibility correction to the SST model, similar to the approach taken by Forsythe et al. ${ }^{12}$, should be further investigated.

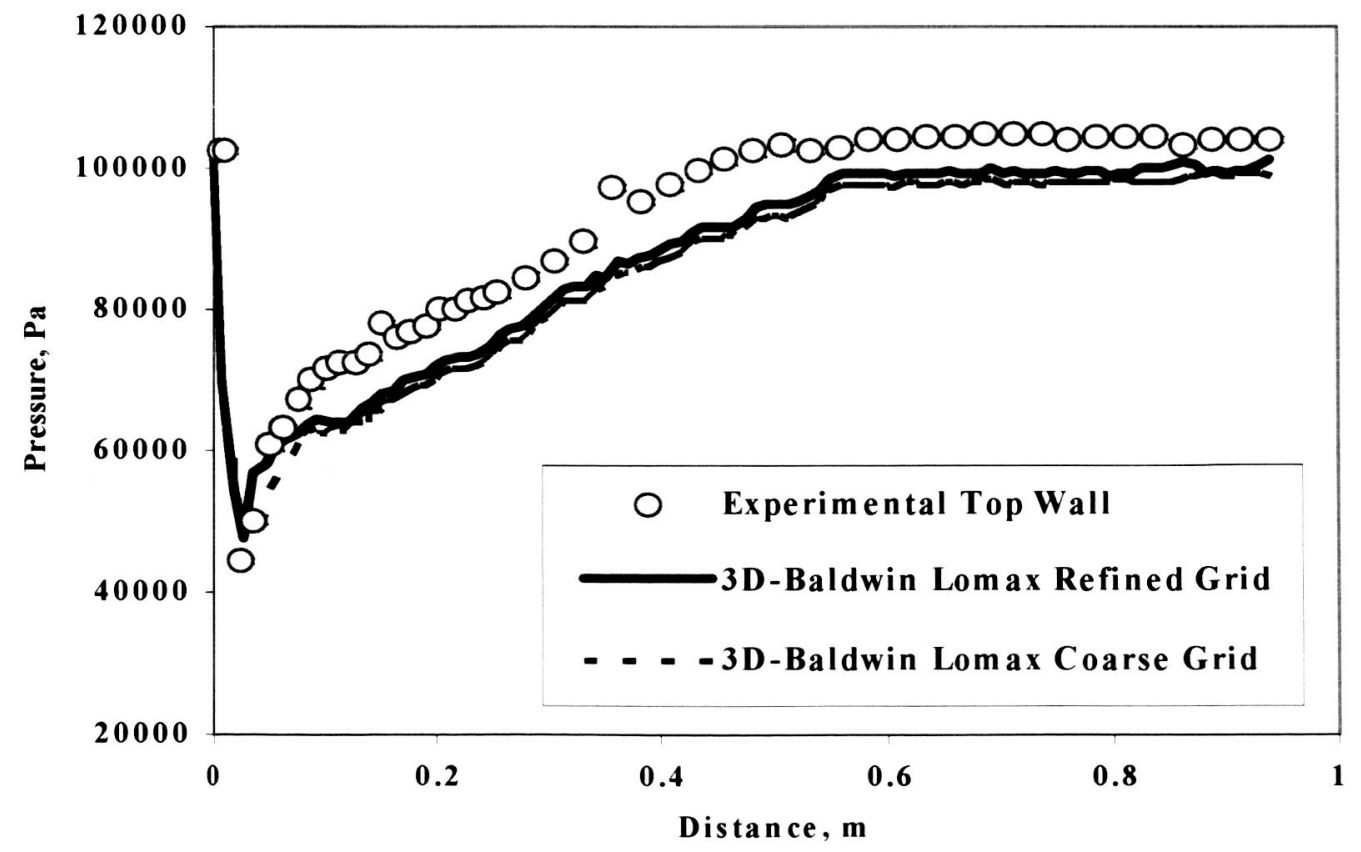

Figure7. 3-D Pressure profiles at centerline of top-wall for ejector chamber pressure $600 \mathrm{psi}$. 


\section{SUMMARY}

A RANS numerical modeling study has been carried out to investigate the flow characteristics of the nonaxisymmetric strut/ejector-based system. In the flow regions dominated by stream mixing, the compressibility correction was found to be necessary to capture the decreased spreading rate of compressible flow streams. The wall functions used in the $k-\varepsilon$ model, even with compressibility correction, cannot reproduce wall-dominated phenomena, such as pressure recovery. 3D modeling with wall-resolving turbulence models is required to resolve the complex strut-base separation features. Full 3D parallel computations, incorporating compressibility corrected SST model are underway. These results will form the basis of a future communication.

\section{ACKNOWLEDGEMENTS}

This work was supported by NASA Grant NCC8-200 with NASA MSFC with Mary Trawek as the COTR for NASA and Dr. Clark W. Hawk as Principal Investigator for the UAH Propulsion Research Center.

\section{REFERENCES}

${ }^{1}$ Balasubramanyam, M. S., Landrum, D. B., Chen, C. P., and Lineberry, D., "Numerical Investigation of Cold Flow Non-Axisymmetric Ejectors", AIAA Paper 2005-1209, 2005.

${ }^{2}$ Bartosiewicz, Y., Aidoun, Z., Desevaux, P. and Mercadier, Y., "Numerical and Experimental Investigations on Supersonic Ejectors," Int. J. Heat and Fluid Flow, Vol. 26, pp. 56-70, 2005.

${ }^{3}$ Chen, Y. S., Chen, C. P. and Wei, H., "Numerical Analysis of Hypersonic Turbulent Film Cooling Flows," AIAA Paper 1992-2767, 1992.

${ }^{4}$ Shyy, W., Thakur, S. S., Ouyang, H., Liu, J. and Blosch, E., Computational Techniques for Complex Transport Phenomena, Cambridge University Press, 1997.

${ }^{5}$ Zeman, O., "Dilatational Dissipation: The Concept and Application in Modeling Compressible Mixing Layer", Physics of Fluids A, Vol. 2, No. 2, 1990.

${ }^{6}$ Viegas, J.R., and Rubesin, M.W., "A Comparative Study of Several Compressibility Corrections to Turbulence Models Applied to High Speed Shear Layers", AIAA Paper 1991-1783, 1991.

${ }^{7}$ Sarkar, S., Erlebacher, G., Hussaini, M.Y., and Kreiss H. O., "The Analysis and Modeling of Dilatational Terms in Compressible Turbulence", J. Fluid Mech., Vol. 227, pp 473-493, 1991.

${ }^{8}$ ESI-CFD, "Fastran Theory Manual", Huntsville, Alabama, 2004.

${ }^{9}$ Eklund, D. R., et al., "A Comparative Computational/Experimental Investigation of Mach 2 Flow Over a Rearward-Facing Step", Computers \& Fluids, Vol. 24, No. 5, pp 593-608, 1995.

${ }^{10}$ Balasubramanyam, M.S., Chen, C.P., Habchi, S.D., and Smith, C.E., "Critical Evaluation of Turbulence Models in High Speed Flows with Separation", Huntsville Simulation Conference, Oct. 2004.

${ }^{11}$ Lineberry, D., Smith, N., Landrum, D.B., Hawk, C.W., "Characterization of Cold Flow Non-Axisymmetric Ejectors", AIAA Paper 2003-5231, 2003.

${ }^{12}$ Forsythe, J. R., Hoffmann, K. A. and Suzen, Y. B., "Investigation of Modified Menter's Two-Equation Turbulence Models for Supersonic Applications", AIAA Paper 1999-0873, 1999. 